determined by differences in C-terminal KDEL endoplasmic reticulum retention motifs. J Virol. 2004;78:3710-21. http://dx.doi.org/10.1128/JVI.78.7.3710-3721.2004

Address for correspondence: Martin Beer, Institute of Diagnostic Virology, Friedrich-Loeffler-Institut, Südufer 10, 17489 Greifswald, Germany; email: martin.beer@fli.de

\title{
Highly Pathogenic Swine Getah Virus in Blue Foxes, Eastern China, 2017
}

\section{Ning Shi, Li-Xia Li, Rong-Guang Lu, Xi-Jun Yan, Hao Liu}

Author affiliations: Foshan University, Foshan, China

(N. Shi, H. Liu); Jilin Wildlife Rescue and Rehabilitation Center, Forestry Department of Jilin Province, Changchun, China (L.-X. Li); Chinese Academy of Agricultural Sciences, Changchun (R.-G. Lu, X.-J. Yan)

DOI: https://doi.org/10.3201/eid2506.181983

We isolated Getah virus from infected foxes in Shandong Province, eastern China. We sequenced the complete Getah virus genome, and phylogenetic analysis revealed a close relationship with a highly pathogenic swine epidemic strain in China. Epidemiologic investigation showed that pigs might play a pivotal role in disease transmission to foxes.

$\mathrm{G}$ etah virus (GETV; genus Alphavirus, family Togaviridae) is a mosquitoborne RNA virus that causes death in young piglets, miscarriage in pregnant sows, and mild illness in horses $(1-3)$. Serologic surveys show that the infection might occur in cattle, ducks, and chickens (4); some evidence suggests that GETV can infect humans and cause mild fever $(5,6)$.

In September 2017, twenty-five 5-month-old blue foxes at a farm in Shandong Province in eastern China showed symptoms of sudden fever, anorexia, and depression; 6 of the 25 animals had onset of neurologic symptoms and died on the third day of illness. We collected blood samples from 45 healthy and 25 ill foxes. We subjected the tissue samples from dead animals, including the brains, lungs, spleens, kidneys, livers, intestines, hearts, and stomachs, to hematoxylin and eosin staining. Microscopic examination confirmed the presence of typical lesions in cerebral cortices with mild neuronal degeneration and inflammatory cell infiltration in vessels, as well as severe hemorrhagic pneumonia, congestion, and hemorrhage with a large number of erythrocytes in the alveolar space (Figure) (1). No obvious lesions were found in other organs.

We used supernatants of homogenized brain and lung tissues from each dead fox to inoculate Vero cells, as described previously (7). We observed a cytopathogenic effect within 72 hours. We observed numerous spherical, enveloped viral particles, $\approx 70 \mathrm{~nm}$ in diameter, after negative staining in a transmission electron microscope. To identify potential viral pathogens, we performed reverse transcription PCR (RT-PCR) to detect a panel of viruses, including canine distemper virus, canine parvovirus, canine coronavirus, and canine adenovirus. However, we detected none of these classical endemic viruses.

During the investigation, farmers reported that the foxes had been fed on organs from symptomatic pigs. We therefore tested for the presence of African swine fever virus, pseudorabies virus, porcine reproductive and respiratory syndrome virus, classical swine fever virus, Japanese encephalitis virus, porcine circovirus type 2, porcine circovirus type 3 , porcine cytomegalovirus, and alphavirus by using the primers for those viruses (Appendix Table 2, https://wwwnc.cdc.gov/EID/article/25/6/18-1983-App1. pdf). RT-PCR using universal primers for alphavirus (M2w-cMw3) produced a 434-bp amplicon when we tested all samples from dead foxes. Sanger sequencing of the amplicon and a BLAST search (https://blast.ncbi.nlm.nih.gov/ Blast.cgi) identified the sequence as that of GETV.

To further investigate the epidemic GETV infection, we performed quantitative RT-PCR by using RNA from all fox samples, as described elsewhere (7). Lung samples from all 6 dead foxes were positive, whereas only 2 samples from the remaining 19 ill foxes were also positive. None of the samples from healthy foxes were positive (Appendix Tables 1, 3). We measured serologic neutralizing antibodies by using a GETV isolate from a symptomatic fox, as previously described $(8,9)$. Results showed no neutralizing antibody $(<1: 2)$ in healthy blue foxes (group 1$)$ and variable levels of neutralizing antibodies (1:2 to 1:256) in ill foxes (groups 2-4) (Appendix Table 3). Samples from ill foxes with lower antibody titers had higher copies of RNA (groups 2-4). Spearman correlation analysis revealed a significant negative correlation between antibody titers and viral RNA copy numbers $\left(r^{2}=0.952 ; p<0.01\right)$.

We obtained the complete genome of the novel GETV SD1709 strain (GenBank accession no. MH106780) by using a conventional RT-PCR method (10). SD1709 genome sequence comparisons showed high identity with the porcine GETV strain (HuN1) at the nucleotide (99.6\%) and deduced amino acid (99.7\%-99.8\%) sequences (Appendix Table 4). Furthermore, phylogenetic analysis of the 

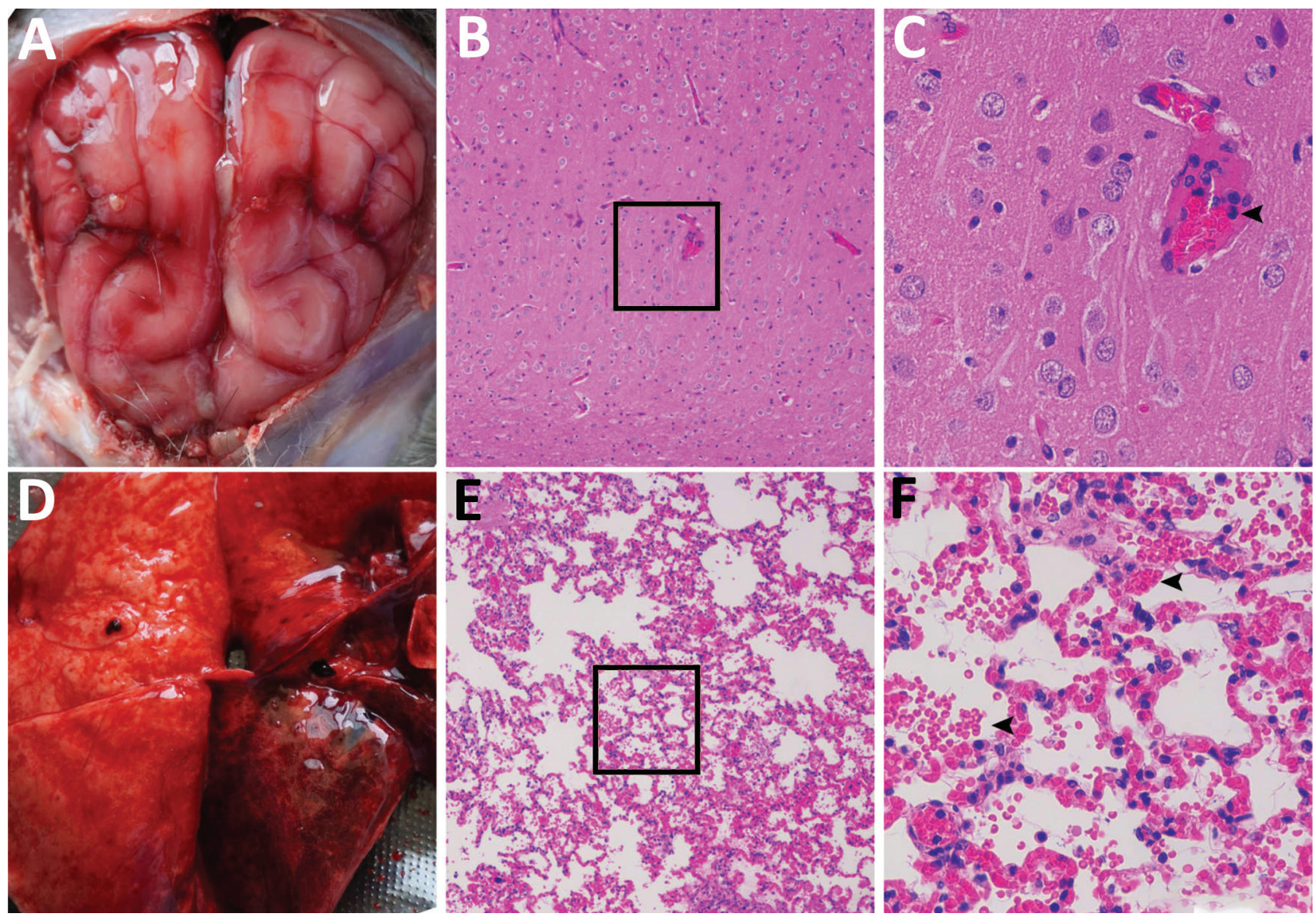

Figure. Dissected brain and lung of a dead fox, collected in 2017 in Shandong Province, eastern China, and histopathologic examination of samples using hematoxylin and eosin staining. A) Brain, showing congestion in the meninx. B) Histologic view of meninx, showing mild neuronal degeneration and inflammatory cell infiltration in vessels. Original magnification $\times 100$. Box indicates area enlarged in panel C. C) A higher magnification view (original magnification $\times 400$ ) of lesions in panel B, showing inflammatory cell infiltration in a vessel (arrow). D) Lung tissue, showing extensive congestion and hemorrhage. E) Histologic view of lung tissue, showing congestion, hemorrhage, or both, with many erythrocytes in the alveolar space. Original magnification $\times 100$. Box indicates area enlarged in panel F. F) A higher magnification view (original magnification $\times 400$ ) of tissue lesions in panel $\mathrm{E}$, showing erythrocytes in the alveolar space (arrows).

complete genome and structural protein E2 gene indicated that the SD1709 strain was most similar to the recent epidemic HuN1 strain, which had caused large numbers of piglet deaths, stillbirths, and fetal mummies in southern China in 2017 (1) (Appendix Figures 1, 2).

We also detected GETV infection in pig serum samples and in mosquitoes (Culex tritaeniorhynchus, Anopheles sinensis, and Armigeres subalbatus) collected in the same region. The infection rate in pigs detected by quantitative RT-PCR was $20.0 \%(4 / 20)$ and by serum neutralization was $75.0 \%(15 / 20)$. The minimum infection rate in mosquitoes was $\approx 1.09 \%$; C. tritaeniorhynchus mosquitoes had a higher minimum infection rate $(2.31 \%)$ compared with other mosquito species $(0-0.80 \%)$. These results suggest that pigs and C. tritaeniorhynchus mosquitoes might play a role in transmitting highly pathogenic GETV to captive foxes in this region (Appendix Tables 5, 6).
In China, the disease caused by GETV has only been reported in pigs in Hunan Province, although the virus has been detected in mosquitoes in $>10$ provinces $(1,4)$. Our study provides evidence that GETV can cause lethal infection in blue foxes. Investigation of transmission routes for GETV in animals might help to prevent outbreaks of GETV disease in China.

\section{Acknowledgments}

We thank Ningyi Jin and Quan Liu for their discussions and suggestions.

This work was supported by the National Key Research and Development Program of China (grant no. 2017YFD0500104), the National Natural Sciences Foundation of China (grant no. 31802199), and the Key Laboratory for Preventive Research of Emerging Animal Diseases in Foshan University (grant no. KLPREAD201801-07). 


\section{About the Author}

Dr. Shi is a researcher at the School of Life Sciences and Engineering, Foshan University, Foshan, Guangdong Province, China. His research interests include emerging mosquitoborne infectious diseases.

\section{References}

1. Yang T, Li R, Hu Y, Yang L, Zhao D, Du L, et al. An outbreak of Getah virus infection among pigs in China, 2017. Transbound Emerg Dis. 2018;65:632-7. http://dx.doi.org/10.1111/tbed.12867

2. Yago K, Hagiwara S, Kawamura H, Narita M. A fatal case in newborn piglets with Getah virus infection: isolation of the virus. Nippon Juigaku Zasshi. 1987;49:989-94. http://dx.doi.org/10.1292/ jvms1939.49.989

3. Nemoto M, Bannai H, Tsujimura K, Kobayashi M, Kikuchi T, Yamanaka T, et al. Getah virus infection among racehorses, Japan, 2014. Emerg Infect Dis. 2015;21:883-5. http://dx.doi.org/10.3201/ eid2105.141975

4. Li Y, Fu S, Guo X, Li X, Li M, Wang L, et al. Serological survey of Getah virus in domestic animals in Yunnan Province, China. Vector Borne Zoonotic Dis. 2019;19:59-61. http://dx.doi.org/10.1089/ vbz.2018.2273

5. Li XD, Qiu FX, Yang H, Rao YN, Calisher CH, Calisher CH. Isolation of Getah virus from mosquitos collected on Hainan
Island, China, and results of a serosurvey. Southeast Asian J Trop Med Public Health. 1992;23:730-4.

6. Marchette NJ, Rudnick A, Garcia R. Alphaviruses in peninsular Malaysia: II. Serological evidence of human infection. Southeast Asian J Trop Med Public Health. 1980;11:14-23.

7. Shi N, Liu H, Li LX, Hu B, Zhang L, Zhao CF, et al. Development of a TaqMan probe-based quantitative reverse transcription PCR assay for detection of Getah virus RNA. Arch Virol. 2018;163: 2877-81. http://dx.doi.org/10.1007/s00705-018-3927-2

8. Kuwata R, Shimoda H, Phichitraslip T, Prasertsincharoen N, Noguchi K, Yonemitsu K, et al. Getah virus epizootic among wild boars in Japan around 2012. Arch Virol. 2018;163:2817-21. http://dx.doi.org/10.1007/s00705-018-3897-4

9. Bannai H, Nemoto M, Niwa H, Murakami S, Tsujimura K, Yamanaka T, et al. Geospatial and temporal associations of Getah virus circulation among pigs and horses around the perimeter of outbreaks in Japanese racehorses in 2014 and 2015. BMC Vet Res. 2017;13:187. http://dx.doi.org/10.1186/s12917-017-1112-6

10. Li YY, Liu H, Fu SH, Li XL, Guo XF, Li MH, et al. From discovery to spread: the evolution and phylogeny of Getah virus. Infect Genet Evol. 2017;55:48-55. http://dx.doi.org/10.1016/ j.meegid.2017.08.016

Address for correspondence: Hao Liu, Foshan University, School of Life Sciences and Engineering, 18 Jiangwan First Rd, Chancheng District, Foshan, Guangdong 528000, China; email: liuhao_lh@hotmail.com

\section{Correction: Vol. 25, No. 5}

The affiliation for Marie E. Killerby was listed incorrectly in Risk Factors for MERS-CoV Seropositivity among Animal Market and Slaughterhouse Workers, Abu Dhabi, United Arab Emirates, 2014-2017 (A. Khudhair et al.). Dr. Killerby is affiliated only with the Centers for Disease Control and Prevention (Atlanta, GA, USA). The article has been corrected online (https://wwwnc.cdc.gov/eid/ article/25/5/18-1728_article).

\section{Get the content you want delivered to your inbox.}

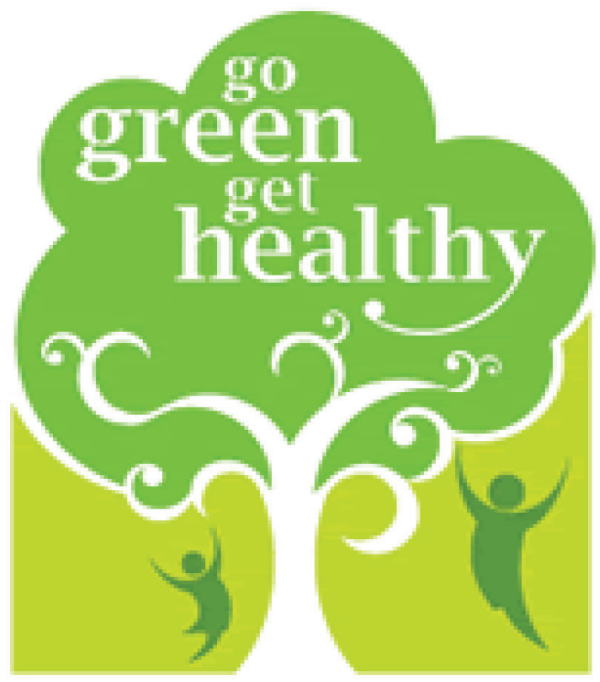

- Table of Contents

- Podcasts

- Ahead of Print articles

- CME

- Specialized Content

\section{Online subscription: wwwnc.cdc.gov/eid/subscribe/htm}

